\title{
LOW COST APPLICATION MOTION HAND GESTURE CONTROLLER USING ADAPTABLE SENSORS
}

\author{
Mr. Akshay Kumar Mishra \\ MCA Scholar,School of CS \& IT, Dept of MCA, JAIN(Deemed-to-be) University, Bangalore
}

Article DOI: https://doi.org/10.36713/epra4398

\begin{abstract}
Today we are surrounded by innovative technology and it is evolving day by day. Among these technologies, the hand gesture is one of such techniques that helps humans to interact with the computer and control its functions using some pre-defined gestures. Usage of hand gesture technology eliminates the use of hardware we use traditionally such as mouse and keyboard as our interaction with the computer is going to be contactless making it a more affordable approach. This technology uses ultrasonic sensors that work on the principle of ultrasonic waves produced by the gesture making the interaction process faster. This technology is thus time-saving, cost-effective, and efficient. The hardware requirement of this technology consists of Arduino UNO board, low in cost sensors, and a personal computer making it highly affordable. This technology not only brings advancement human-computer interaction but also speeds up the process, making it a productive approach to choose. [4]
\end{abstract}

KEYWORDS- Arduino, Ultrasonic Sensors, Leap motion, Gesture, Laptop, PySerial, PyAutoGUI, Python,

\section{INTRODUCTION}

In this world, between technologies like automation, artificial intelligence, data analytics, etc., the use of gesturecontrolled computers and laptops is getting very famous. In this technique, one can control the functions of their computers or laptops by only a wave of their hand. This technology is called leap motion. It may sound simple and interesting, but the system used in this technology is not very affordable. The proposed model uses the power of Arduino and Python which not only makes it a cheaper and affordable approach to leap motion, but it is also easier to build.

The proposed model uses two ultrasonic sensors which help to locate the position of our hand. The equipment used in making this model is what makes it a highly affordable choice over the existing one. In this model, we can control a media player (VLC) based on the gesture type and the position of our hands. The media player used in this model is for the demonstration purpose, but once the working mechanism of the model is understood, one can follow the same procedure to control any desired function or operation of their system however they want by just modifying a few lines of code.

The advantage of using this approach over the existing one is the cost-effectiveness and the simplicity of the concept that this model offers. In this approach, the distance value between the sensors and the hands is collected, it is then analyzed resulting in the action being performed. The actions that need to be performed are carried out with the help of Python PyAutoGUI library. The Arduino commands are then sent to the computer through a serial port (USB). These data are then analyzed using Python codes which are written in Python that is installed on the computer.

\section{A. Motivation}

Automation of the existing technology is my motive in this project. This project is not only advanced but also costeffective when compared to the traditional approach. With very low-cost IoT devices we can achieve this technique. If we will buy a laptop with leap motion technology or gesture deduction technology it will cost us a huge amount of money as these devices are very costly. So with this approach we can make our own gesture control laptop or system at a very low cost.

\section{B. Benefits of the projected system}

- A number of functions of computers can be operated by using ultrasonic sensors.

- Using this technique, it is easy to interact with the computer and there are no language barrios

- This technique may be very useful for those who do not know the functionality of computer

- Using this technique, you can control your laptop without touching it physically 


\section{EPRA International Journal of Research and Development (IJRD) Volume: 5 | Issue: 5 | May 2020 \\ - Peer Reviewed Journal}

- We can control our laptop with a small distance and it can help to control laptops in conference room presentations.

\section{LITERATURE REVIEW}

- In this paper the author had told about how gesture has become an important means of communication in the physical world interaction with machines become very important and how we can control some of our application with a gesture with the help of IoT devices such as Arduino UNO and ultrasonic sensors[1]

- This paper states that problem cause by keyboard and mouse sometimes can be helpful with this gesturebased application as it is very easy to use. It uses very simple gesture to control the video to the people don't have to learn machine-like skills only the people have to remember the type of gesture to control the application[2]

- In this paper the author proposed that lots of techniques are there to interact with humans and machines and one of them is the hand gesture technique in this technique hand gesture is used to control the machine instead of mouse and keyboard. It is an effective and faster technique. To determine the user hand an ultrasonic sensor is used. By using this technique there is no need for a physical connection between the humans and the machine.[3]

- In this paper the author states that gesture is an expression of emotion and physical behavior. Gesture can be used as a tool that can be used to interact and communicate between the machine and the computer. The hand gesture is different from the traditional hardware-based method gesture that has been proposed has different types of concepts like SVM, neural network, HMM, Arduino coding, etc.[4]

\section{WORKING}

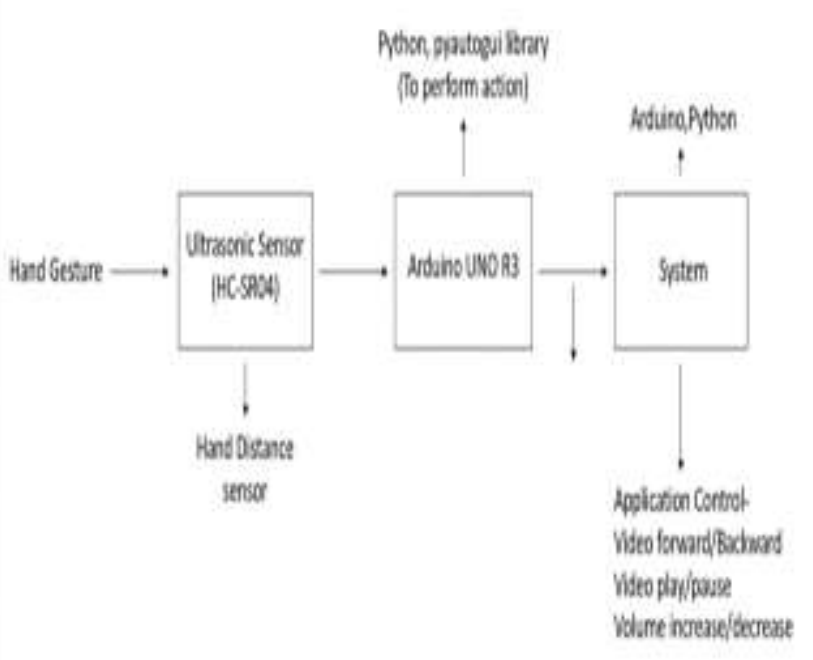

Figure 1.0 Architecture
Here we are able to see that there are two Ultrasonic Sensors(HC-SR04) are connected to the Arduino UNO R3 board is connected to the laptop through USB cable. The ultrasonic sensors accommodate the transmitter and receiver. The transmitter emits ultrasonic waves. The waves get better when comes in reality with any obstacle (example hand) and therefore the sensors catch it. supported the gap of the article is set. Then the information is received by the Arduino and check for the particular keyword within the code in keeping with the gap that's to be the python code. Python catches the keyword and creates a virtual keystroke of hotkeys. The ultrasonic sensors measure the gap between the hand and therefore the sensors and supported the gap it performs an action like volume increase or decrease and the same thing happens with play or pause of the video.

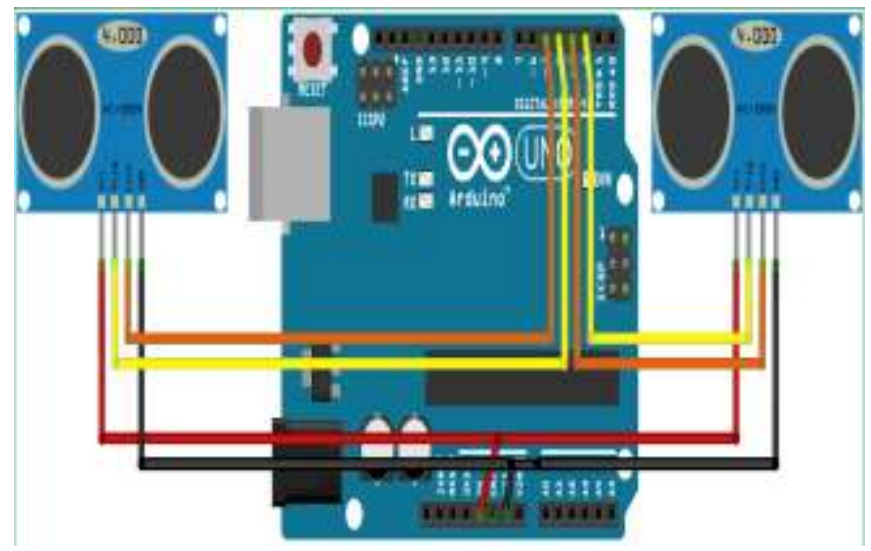

Fig 1.1 circuit diagram[12]

As we can see in Fig 1.1 there are two ultrasonic sensors that is connected to Arduino UNO board and the board is connected with the laptop with the help of USB cable. The VCC pins of both the sensors are connected with the $5 \mathrm{~V}$ output port in the Arduino board. The grounding of the sensors is attached to the GND. The echo and trigger of the sensors are connected with the Arduino board according to the code written. The ultrasonic sensors emit ultrasonic waves from the transmitter present on it the waves it. The waves hit the object that is present in front of the sensors and received by the receiver present inside the sensors. Based on the intensity the distance of the object is going to be determined. Then this data is sent to the Arduino and looks for the keyword that is present in the code then it is sent to the python code that is running in the background. Python takes the keyword and generates the hotkeys for the VLC media player. This process will keep on continuing to control the media player either audio or video file. The ultrasonic sensors read the gesture produced by the hand and the distance from the hand to the sensors. If we will bring our right hand near to the sensors the volume will decrease and if we will take our hand back from the sensors, then the sound will decrease, the same action will be done with the left sensors it will perform forward and backward of the video. 


\section{SJIF Impact Factor: 7.001| ISI I.F.Value:1.241| Journal DOI: 10.36713/epra2016 \\ ISSN: 2455-7838(Online) \\ EPRA International Journal of Research and Development (IJRD) \\ - Peer Reviewed Journal}

\section{A. HARDWARE USED}

1. Arduino - It is an open-source electronics platform based on easy to use hardware and software. It is a microcontroller you can do programming on it it's as it supports Arduino programming language and will be able to perform some task with the help of Arduino as shown in figure 2.0 [1]

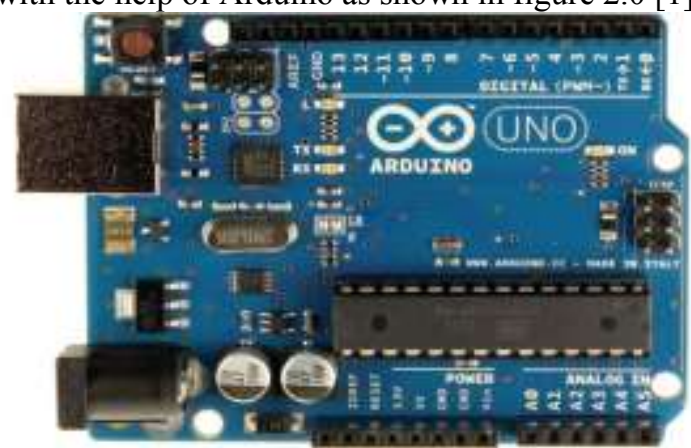

Fig 2.0 Arduino UNO

2. Ultrasonic sensors(HC-SR04)- it is a type of sensors that are used to deduct the distance of an object using ultrasonic waves. It consists of transistors and receivers the transistors emit the ultrasonic waves and after hitting the object it will be cached back by the receiver present in the sensors. Ultrasonic sensor is shown in figure 2.1 [2]

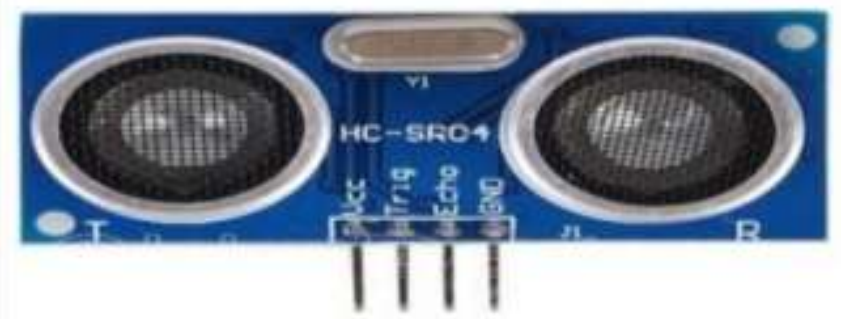

Fig 2.1 Ultrasonic Sensor

\section{B. SOFTWARE USED}

1. Arduino IDE - The Arduino Integrated Development Environment (IDE) is an application that is platform-independent and is written using $\mathrm{C}$ and $\mathrm{C}++$. This IDE is used to write programs for Arduino compatible boards.

2. Python IDLE - A Python IDE (or Integrated Development Environment) is a program dedicated to software development that uses Python codes to operate.

3. PySerial library - It is a library used for connecting serial ports with various devices such. It is also used to connect to remote serial ports via RFC 2217.

4. PyAutoGUI library - PyAutoGUI is a library used to control the hardware such as mouse and keyboard. We are using it to perform actions.
Table N0: 1

\begin{tabular}{|c|c|c|}
\hline Hand gesture & \multicolumn{2}{|c|}{ Output made by system } \\
\hline & video & audio \\
\hline Right hand push in & Volume down & Volume down \\
\hline $\begin{array}{c}\text { Right hand pull } \\
\text { out }\end{array}$ & Volume up & Volume up \\
\hline Left hand push in & forward & forward \\
\hline Left hand pull out & backward & backward \\
\hline Both hand straight & Play & Play \\
\hline Both hand straight & pause & pause \\
\hline
\end{tabular}

\section{IMPLEMENTATION}

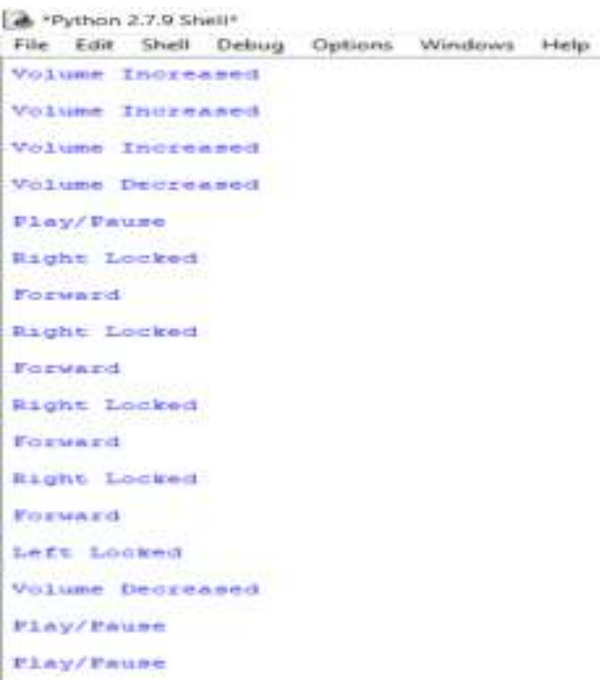

Fig 3.1 python GUI output screen

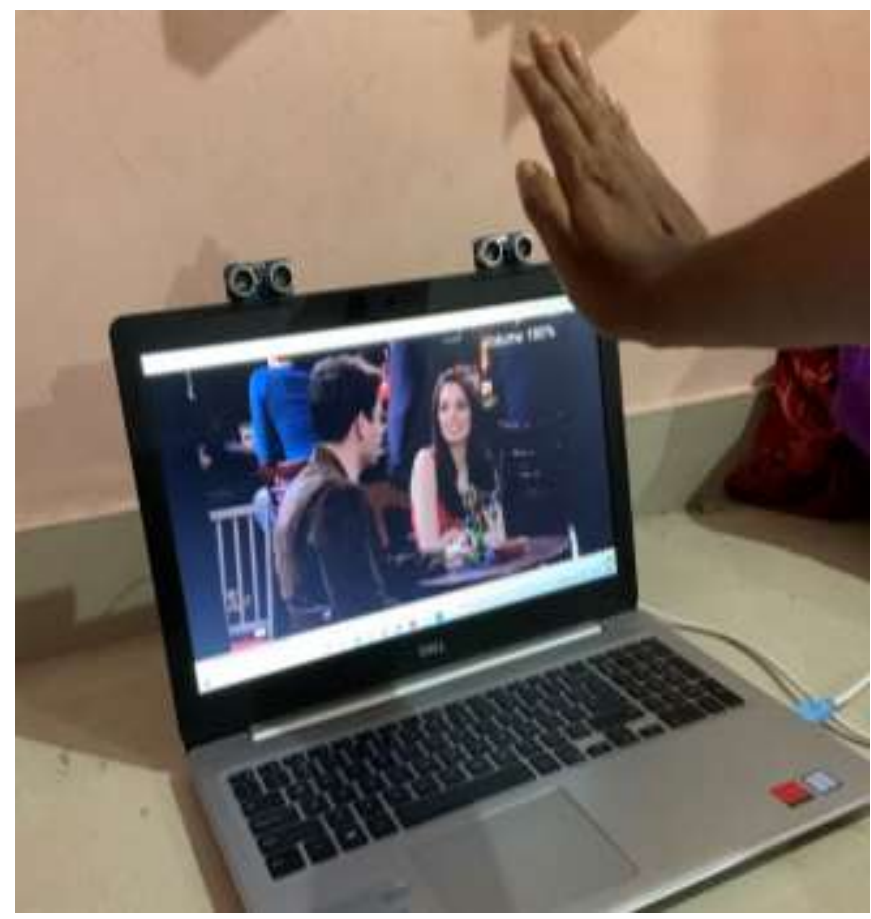

Fig 3.2 Volume up output 


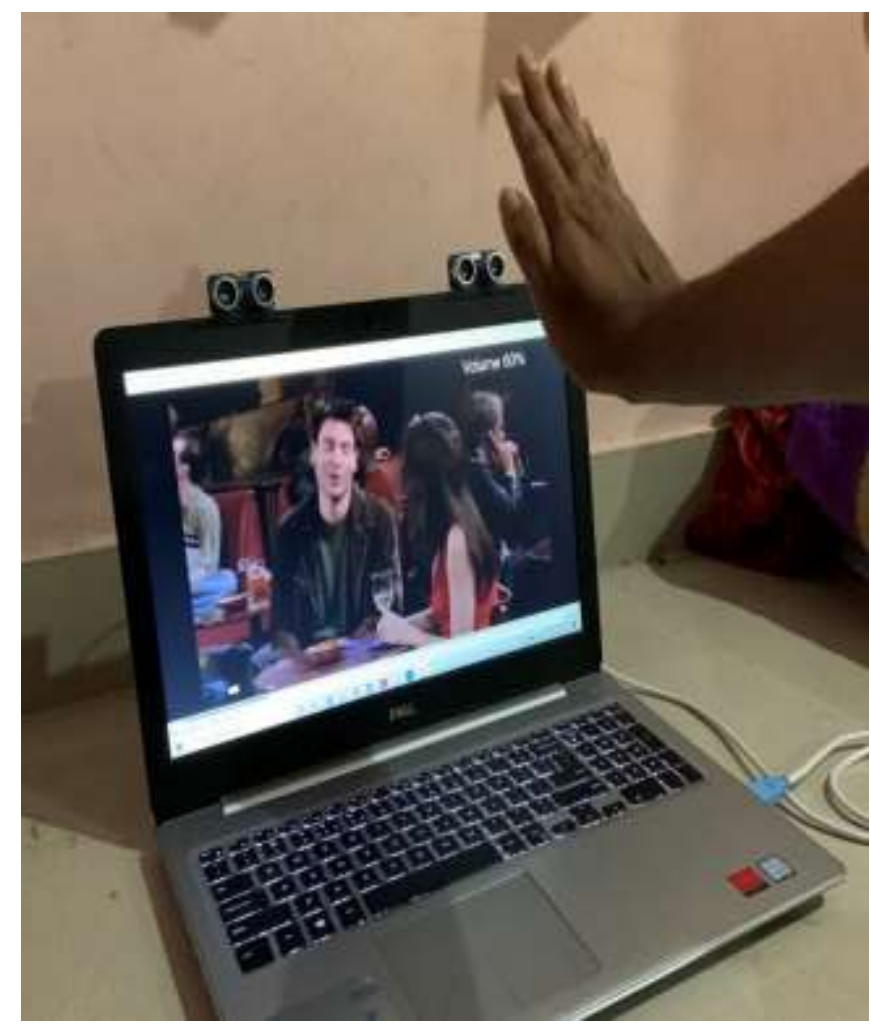

Fig 3.3 Volume down output

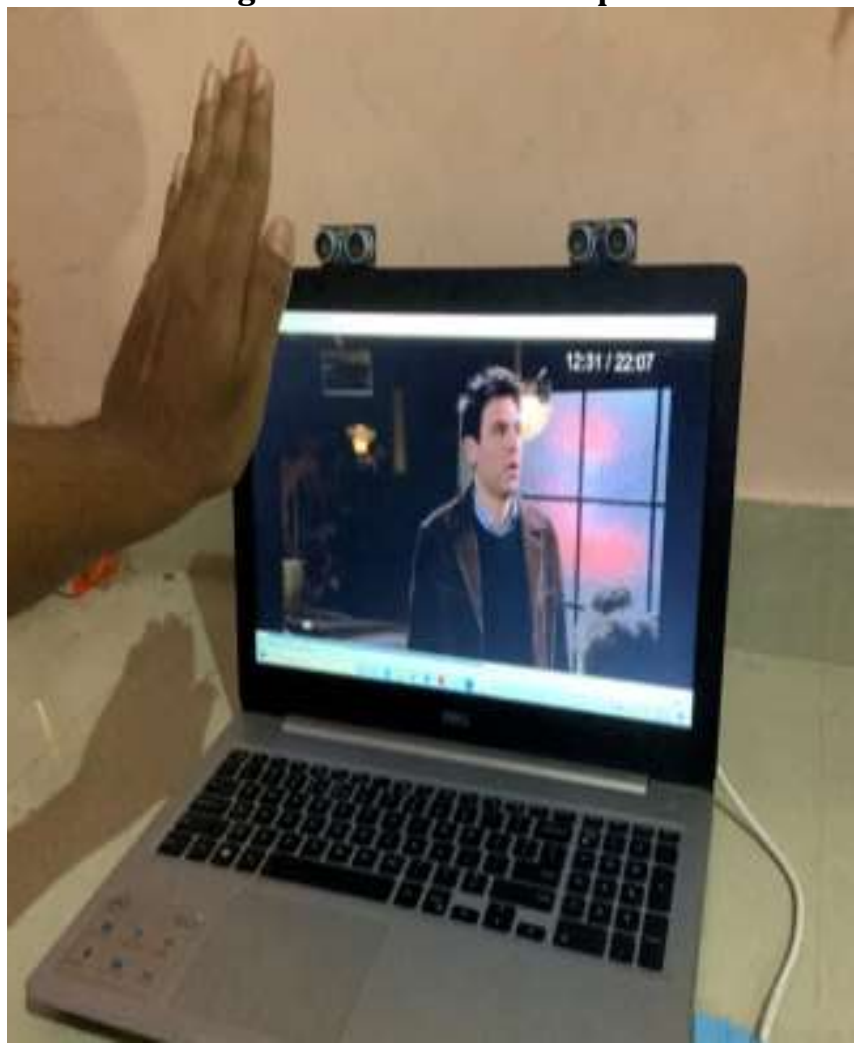

Fig 3.4 Forward output

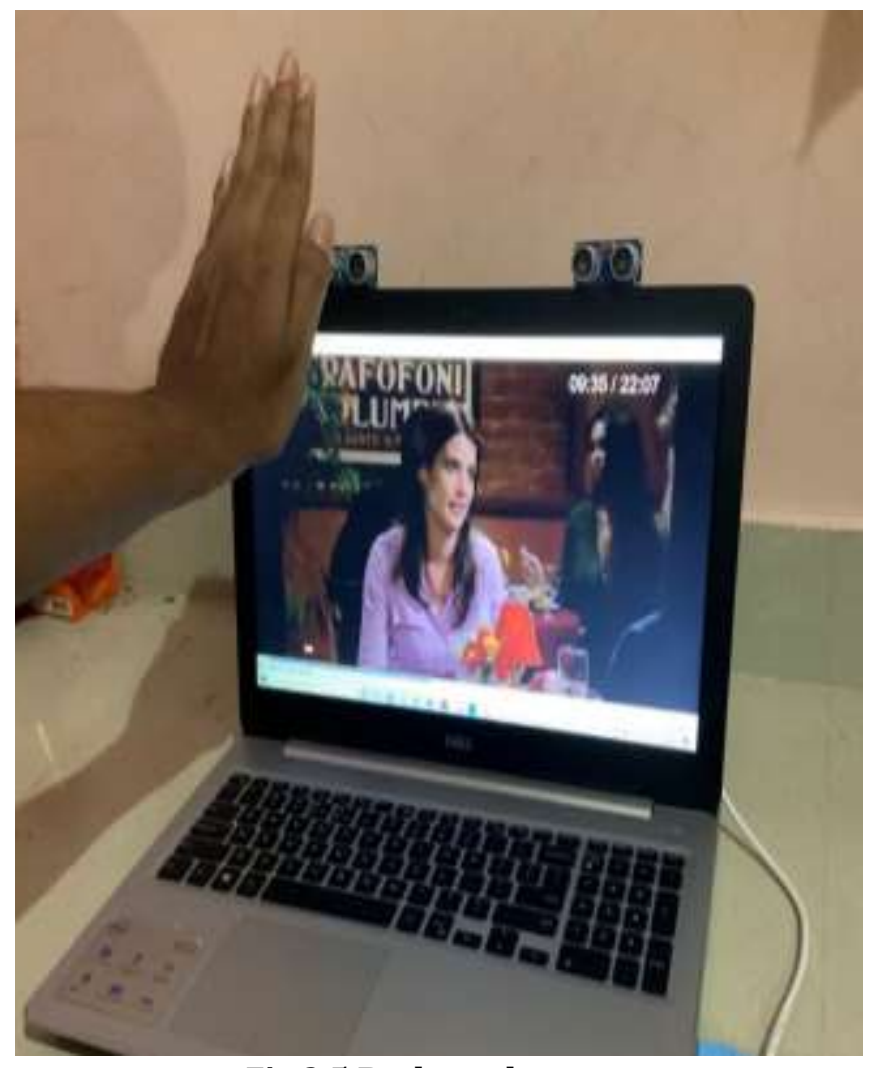

Fig 3.5 Backward output

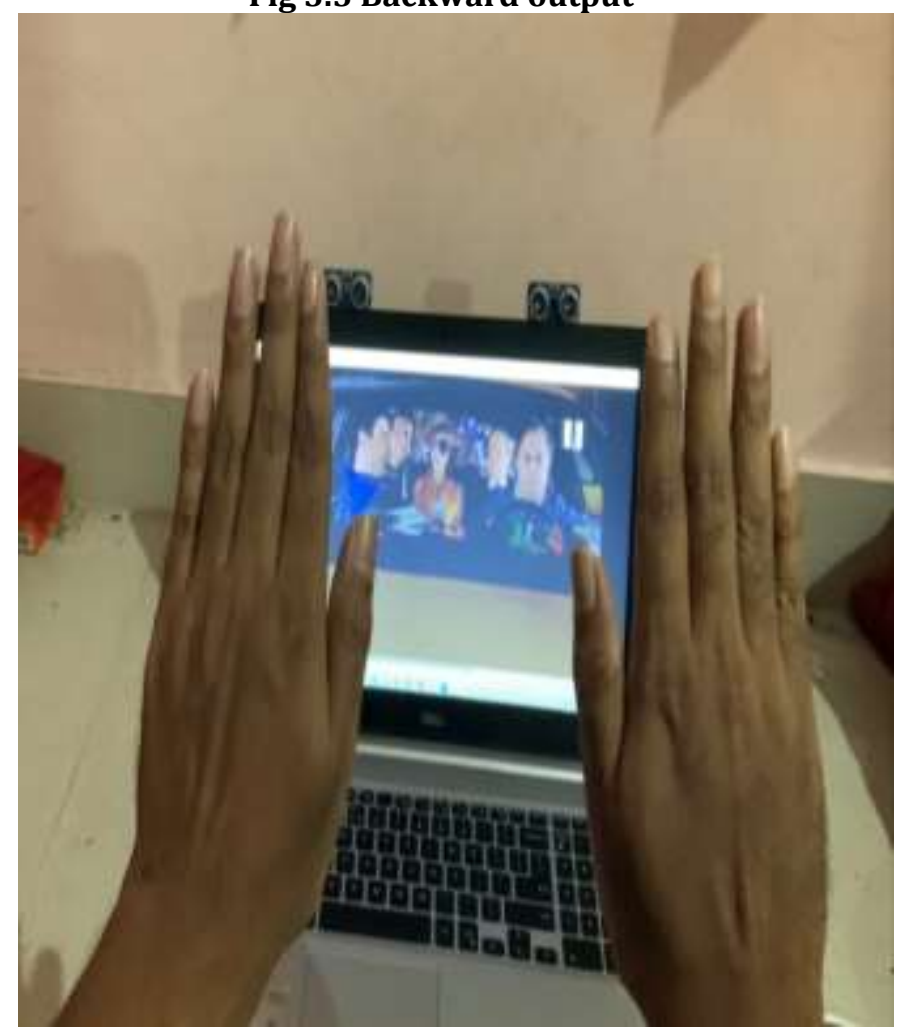

Fig 3.6 Play output 


\section{SJIF Impact Factor: 7.001| ISI I.F.Value:1.241| Journal DOI: 10.36713/epra2016 \\ ISSN: 2455-7838(Online) \\ EPRA International Journal of Research and Development (IJRD) \\ Volume: 5 | Issue: 5 | May 2020 \\ - Peer Reviewed Journal}

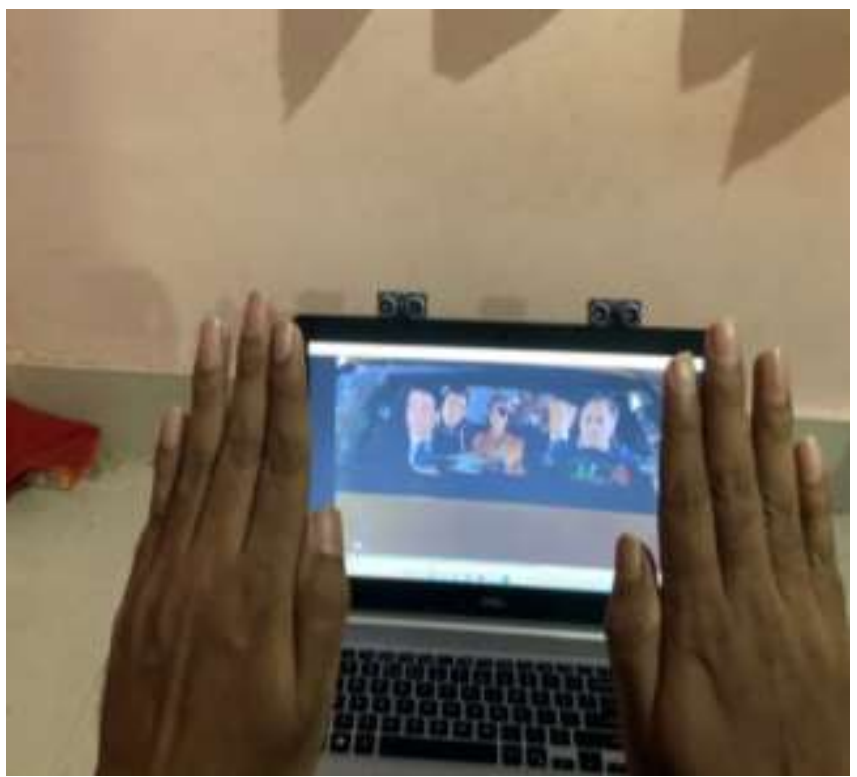

Fig 3.7 Pause output

\section{CONCLUSION AND FUTURE DEVELOPMENT}

\section{A. CONCLUSION}

The gesture control system uses two ultrasonic sensors, Arduino UNO, and a system to carry the operation of video player controller. Its main motive is to reduce the effort of interacting with the system with the input devices and using simple hand gestures instead of that. It increases interactivity with computers. This type of technology can be used in giving presentations, classrooms for easier and interactive learning, gaming, etc. with this kind of techniques, we can also make other gesture reorganization system like opening taps without touching it only waving hand in front of it to open it, using hand wash without touching it by hand gesture and many more.

\section{B. FUTURE DEVELOPMENT}

1. This project can be further implemented on many other gesture controls machines

2. We can control many applications with this by just changing some of the codes

3. We can integrate this type of module for many applications like browsers, designing and editing applications, gaming, etc.

4. The knowledge is ever-expanding and so are the problems that mankind strives to solve.

\section{REFERENCES}

[1] octopart, "Octopart," [Online]. Available: https://octopart.com/a000066-arduino-21222240.

[2] potentiallabs, "Potentiallabs," [Online]. Available: https://potentiallabs.com/cart/HC-SR04-Ultrasonic-

Proximity-sensor.

[3] R. Mukherjee, P. swethan, R. Pasha and S. s. Rawat, "HAND GESTURE CONTROLLED LAPTOP USING ARDUINO," International Journal of Management, Technology And Engineering, p. 7, 2018.

[4] G. A. kumar and B. s. , "Gesture controlled video playback," INTERNATIONAL JOURNAL OF CURRENT ENGINEERING AND SCIENTIFIC RESEARCH (IJCESR), $p$. 4, 2018.

[5] S. Mishra and T. Dhikhi, "Gesture control keyboard," International Journal of Research in Engineering, Science and Management, p. 5, 2018.

[6] R. Remolda and D. A. Kumar, "A study on controlling a computer with hand gesture," International Journal of Computer Science and Mobile Computing, p. 4, 2019.

[7] M. S. Khasale, "Computer Control with Hand Gestures Using Ultrasonic," International Journal of Innovations \& Advancement in Computer Science, p. 7, 2018.

[8] Instructables, "Amazing Control Computer Using Hand Motion and Arduino," [Online]. Available: https://www.instructables.com/id/Amazing-ControlComputer-Using-Hand-Motion-and-Ard/.

[9] H. Iqbal, "appuals," 30 september 2019. [Online]. Available: https://appuals.com/how-to-make-arduino-based-handgesture-control-of-your-computer/.

[10] S. B. Rumy, "Technical Ustad," 9 January 2018. [Online]. Available: https://technicalustad.com/control-your-pc-withhand-gesture-using-arduino/.

[11] R. "electronicshub," 15 november 2017. [Online]. Available: https://www.electronicshub.org/arduino-based-hand-gesturecontrol-computer/.

[12] Create.arduino, "Project hub," 28 december 2017. [Online]. Available: https://create.arduino.cc/projecthub/smarttech/amazing-control-computer-using-hand-motion-andarduino-d933f1.

[13] A. Raj, "circuitdiges," 24 october 2017. [Online]. Available: https://circuitdigest.com/microcontroller-projects/controlyour-computer-with-hand-gestures.

[14] A. Raj, "Circuitdigest," 24 October 2014. [Online]. Available: $\quad$ https://circuitdigest.com/microcontrollerprojects/control-your-computer-with-hand-gestures. 